\title{
A nomogram based on age, prostate-specific antigen level, prostate volume and digital rectal examination for predicting risk of prostate cancer
}

\author{
Ping Tang ${ }^{1,2}$, Hui Chen ${ }^{3}$, Matthew Uhlman ${ }^{4}$, Yu-Rong Lin ${ }^{2}$, Xiang-Rong Deng ${ }^{2}$, Bin Wang ${ }^{2}$, Wen-Jun Yang ${ }^{2}$ \\ and $\mathrm{Ke}-\mathrm{Ji} \mathrm{Xie}{ }^{2}$
}

Nomograms for predicting the risk of prostate cancer developed using other populations may introduce sizable bias when applied to a Chinese cohort. In the present study, we sought to develop a nomogram for predicting the probability of a positive initial prostate biopsy in a Chinese population. A total of 535 Chinese men who underwent a prostatic biopsy for the detection of prostate cancer in the past decade with complete biopsy data were included. Stepwise logistic regression was used to determine the independent predictors of a positive initial biopsy. Age, prostate-specific antigen (PSA), prostate volume (PV), digital rectal examination (DRE) status, \% free PSA and transrectal ultrasound (TRUS) findings were included in the analysis. A nomogram model was developed that was based on these independent predictors to calculate the probability of a positive initial prostate biopsy. A receiver-operating characteristic curve was used to assess the accuracy of using the nomogram and PSA levels alone for predicting positive prostate biopsy. The rate for positive initial prostate biopsy was $41.7 \%(223 / 535)$. The independent variables used to predict a positive initial prostate biopsy were age, PSA, PV and DRE status. The areas under the receiver-operating characteristic curve for a positive initial prostate biopsy for PSA alone and the nomogram were $79.7 \%$ and $84.8 \%$, respectively. Our results indicate that the risk of a positive initial prostate biopsy can be predicted to a satisfactory level in a Chinese population using our nomogram. The nomogram can be used to identify and counsel patients who should consider a prostate biopsy, ultimately enhancing accuracy in diagnosing prostate cancer.

Asian Journal of Andrology (2013) 15, 129-133; doi:10.1038/aja.2012.111; published online 10 December 2012

Keywords: nomogram; prediction; prostate biopsy; prostate cancer; prostate-specific antigen (PSA); prostate volume (PV); age; digital rectal examination (DRE)

\section{INTRODUCTION}

In 2011, prostate cancer was the most commonly diagnosed cancer and the second leading cause of cancer-related death in men in the United States. ${ }^{1}$ The incidence of prostate cancer in Asian countries has been increasing in recent years, but it is still much lower than in Western countries. ${ }^{2}$ Currently, measurement of prostate-specific antigen (PSA) remains the most commonly used method for diagnosing prostate cancer, although its usefulness as a factor associated with decreasing prostate cancer-specific mortality remains controversial. ${ }^{3,4}$ Nevertheless, the specificity of PSA for predicting prostate cancer is not high. ${ }^{5}$ Nomograms, which integrate multiple independent risk factors for prostate cancer, have been shown to improve prostate cancer detection rates and help reduce unnecessary prostate biopsies. ${ }^{6}$ The Prostate Cancer Prevention Trial (PCPT) and the European Randomised Study of Screening for Prostate Cancer (ERSPC) risk calculators are the best known nomograms for incorporating known risk factors. ${ }^{7}$ Additionally, the incidence of prostate cancer in men from industrialised countries has been shown to be substantially different from that in men from developing countries. ${ }^{2}$ Therefore, nomogram models used to predict the risk of prostate cancer that were generated from male populations in industrialised countries may not be directly applicable to Chinese men. Yoon et al. ${ }^{8}$ used a Korean population to validate the performance of the Prostate Risk Calculator 3 (based on data from the Dutch cohort of the ERSPC). They found that use of the Prostate Risk Calculator 3 in a Korean population would miss $7 \%$ more cancers than in a Caucasian population. We recently reported that baseline PSA and PSA velocity in young Chinese men without prostate cancer differ from those of African American and Caucasian American men. ${ }^{9}$ Furthermore, Zhu et al. ${ }^{10}$ recently reported that the PCPT and ERSPC risk calculators overestimated the probability of predicting prostate cancer and high-grade disease when independently validated using a Chinese population. The existing evidence indicates that evaluation of prostate cancer risk should be tailored for different races. ${ }^{8-10}$ As a result, the development and use of a nomogram for the population from which the nomogram was derived is particularly pertinent. To our knowledge, no nomogram to evaluate the risk of prostate cancer in a Chinese population has been published to date. In this study, we have

${ }^{1}$ Postgraduate Institute, Southern Medical University, Guangzhou 510515, China; ${ }^{2}$ Department of Urology, Guangzhou First Municipal People's Hospital, Guangzhou Medical College, Guangzhou 510180, China; ${ }^{3}$ Department of Urology, Guangdong Provincial Work Injury Rehabilitation Hospital, Guangzhou 510440, China and ${ }^{4}$ Department of Urology, University of lowa, lowa City, IA 52242, USA 
developed a nomogram for predicting the probability of a positive initial prostate biopsy in a Chinese population.

\section{MATERIALS AND METHODS}

\section{Study population}

After institutional review board approval had been obtained, the records of 699 men who had undergone transrectal ultrasound (TRUS)-guided prostate biopsy for the detection of cancer between November 1999 and June 2011 were retrieved from our computer centre. Twelve men who were biopsied more than two times and 152 men without complete data were excluded. This left a total of 535 subjects who were included in the study. Within this cohort, $486(90.8 \%)$ underwent a 13-core biopsy, 28 (5.2\%) underwent a six-core biopsy and $21(3.9 \%)$ underwent a 4-15 core biopsy (not a 6- or 13-core).

\section{Clinical variable evaluation}

PSA was analysed with the Abbott AxSYM PSA (Bayer Corporation, Beijing, China) assay before 2006 and the Roche Elecsys 2010 PSA assay (Roche Diagnostics, Shanghai, China) after 2006. Prostate volume (PV) was determined during TRUS and was calculated using the formula, PV $(\mathrm{ml})=0.52 \times$ anterior-posterior diameter $(\mathrm{cm}) \times$ transverse diameter $(\mathrm{cm}) \times$ superior-inferior diameter $(\mathrm{cm})$. The criteria for a prostatic biopsy were a PSA $\geqslant 10 \mathrm{ng} \mathrm{ml}^{-1}$ or a positive digital rectal examination (DRE; prostatic nodule or firm area). If a man had a PSA between 4 and $10 \mathrm{ng} \mathrm{ml}^{-1}$, the indication for a prostate biopsy was based on clinician assessment or patient preference.

\section{Statistical analysis}

Age, PSA, PV, DRE status, \% free PSA and TRUS findings (low echogenicity in the peripheral zone was defined as positive) were included in the stepwise regression analysis. Because PSA and PV had a nonnormal distribution and a nonlinear effect of these variables was expected, these variables were transformed to the natural logarithm before analysis. Age, PSA, PV and \% free PSA were treated as continuous variables, whereas DRE status and TRUS findings were categorical variables. Variables that did not reach statistical significance at a level of 0.05 were removed from the model in the backward stepwise process.

The independent variables from the final model were used to construct a nomogram to predict the probability of a positive initial biopsy. The nomogram was validated and predictive accuracy calculated using Harrell's Concordance Index C on 100 bootstrapped re-samples. Calibration plots were also generated to assess the discrimination capacity of the nomogram. A receiver-operating characteristic curve was used to evaluate the effectiveness of the nomogram and PSA alone in predicting a positive initial prostate biopsy. Statistical analyses were performed with $\mathrm{R}$ version 2.13.1 (http:// www.r-project.org/).

\section{RESULTS}

The demographic characteristics of the study cohort are shown in Table 1. Of the 535 subjects included in the study, 223 (41.7\%) had a positive initial prostate biopsy. The median and mean diagnostic PSA levels in our study cohort were 18.6 and $91.4 \mathrm{ng} \mathrm{ml}^{-1}$, respectively. The median PSA level in men with a positive initial prostate biopsy was statistically higher than those with a negative biopsy (43.4 $\mathrm{ng} \mathrm{ml}^{-1}$ vs. $13.1 \mathrm{ng} \mathrm{ml}^{-1}, P<0.001$, Mann-Whitney $U$ test). The results of univariate analysis of age, \% free PSA, PV, rate of abnormal DRE and rate of TRUS abnormalities in men with a positive initial biopsy were all significantly different from those in men with a negative biopsy.

Table 1 Characteristics of the study cohort

\begin{tabular}{|c|c|c|c|c|}
\hline Variables & Total & $\mathrm{PCa}$ & Non-PCa & $\mathrm{P}$ \\
\hline No. of subjects (\%) & 535 & $223(41.7)$ & $312(58.3)$ & \\
\hline \multicolumn{5}{|c|}{ Diagnostic PSA (ng ml ${ }^{-1}$ ) } \\
\hline Mean (s.d.) & $91.4(366.6)$ & $176.5(516.5)$ & 28.5 (173.9) & \\
\hline Median (IQR) & $18.6(9.2-47.6)$ & $43.4(18.9-141.9)$ & $13.1(6.8-20.7)$ & $<0.001^{\mathrm{b}}$ \\
\hline Range & $0.0-6282.0$ & $0.0-6282.0$ & 0.0-2986.0 & \\
\hline \multicolumn{5}{|l|}{ Diagnostic \% free PSA } \\
\hline Mean (s.d.) & $18(47)$ & $19(73)$ & $18(11)$ & \\
\hline Median (IQR) & $14(9-20)$ & $11(8-16)$ & $16(10-21)$ & $<0.001^{\mathrm{b}}$ \\
\hline Range & 0-97 & 0-97 & 0-97 & \\
\hline \multicolumn{5}{|c|}{ Age at initial biopsy (year) } \\
\hline Mean (s.d) & $72(9)$ & $73(9)$ & $70(9)$ & \\
\hline Median (IQR) & $72(68-78)$ & 74 (69-79) & $72(66-76)$ & $<0.001^{\text {b }}$ \\
\hline Range & 20-91 & 20-91 & 21-87 & \\
\hline \multicolumn{5}{|l|}{ Prostate volume (ml) } \\
\hline Mean (s.d) & $64(37)$ & $59(38)$ & $67(37)$ & \\
\hline Median (IQR) & $54(38-82)$ & $47(33-70)$ & $60(41-83)$ & $<0.001^{\text {b }}$ \\
\hline Range & 4-319 & 10-262 & 4-319 & \\
\hline \multicolumn{5}{|l|}{ TRUS finding ${ }^{a}$} \\
\hline Positive & 134 & 73 & 61 & $0.019^{c}$ \\
\hline Negative & 401 & 173 & 228 & \\
\hline \multicolumn{5}{|c|}{ Digital rectal examination } \\
\hline Positive & 133 & 87 & 46 & $<0.001^{c}$ \\
\hline Negative & 402 & 140 & 262 & \\
\hline
\end{tabular}

Abbreviations: IQR, interquartile range; PCa, prostate cancer; PSA, prostate-specific antigen; TRUS, transrectal ultrasound.

a Low-echogenicity in the peripheral zone of the prostate was defined as 'positive'; other findings were defined as 'negative'.

${ }^{\mathrm{b}}$ Mann-Whitney $U$ test.

${ }^{\mathrm{c}}$ Chi-square test. 
Table 2 The predictors of a positive initial biopsy in multivariate analysis

\begin{tabular}{|c|c|c|c|c|}
\hline \multirow{2}{*}{ Variables } & \multirow{2}{*}{ Odds ratio } & \multicolumn{2}{|c|}{$95 \% \mathrm{Cl}$} & \multirow{2}{*}{$P$} \\
\hline & & Lower & Upper & \\
\hline Age at biopsy & 1.0 & 1.0 & 1.1 & 0.026 \\
\hline $\log (P S A)$ & 9.9 & 6.1 & 16.0 & $<0.001$ \\
\hline $\begin{array}{l}\text { Log (prostate } \\
\text { volume) }\end{array}$ & 0.06 & 0.02 & 0.16 & $<0.001$ \\
\hline $\begin{array}{l}\text { Diagnostic \% free } \\
\text { PSA }\end{array}$ & 7.1 & 0.1 & 366.6 & 0.331 \\
\hline \multicolumn{5}{|l|}{ DRE } \\
\hline Negative & Reference & & & \\
\hline Positive & 2.8 & 1.7 & 4.7 & $<0.001$ \\
\hline \multicolumn{5}{|l|}{ TRUS finding ${ }^{a}$} \\
\hline Negative & Reference & & & \\
\hline Positive & 1.5 & 0.7 & 3.4 & 0.302 \\
\hline
\end{tabular}

Abbreviations: $\mathrm{Cl}$, confidence interval; $\mathrm{PCa}$, prostate cancer; PSA, prostate-specific antigen; TRUS, transrectal ultrasound; DRE, digital rectal examination.

a Low-echogenicity in the peripheral zone of the prostate was defined as 'positive'; other findings were defined as 'negative'.

In a multivariate analysis, only age, PSA, LogPSA, and LogPV were found to be independent predictors of a positive initial prostate biopsy (Table 2). The logistic regression model yielded the equation below:

Positive initial prostate biopsy probability equation for Chinese population $=$ $\mathrm{e}^{-1.163+0.033 \mathrm{Age}+1.032 \mathrm{DRE}-2.821 \operatorname{LogPV}+2.292 \mathrm{LogPSA}}$

$1+\mathrm{e}^{-1.163+0.033 \mathrm{Age}+1.032 \mathrm{DRE}-2.821 \operatorname{LogPV}+2.292 \operatorname{LogPSA}}$

Using the information from multivariate regression analysis, a nomogram model was developed that allows the calculation of an individual patient's risk for a positive initial prostate biopsy (Figure 1). Figure 2 shows validation of the nomogram. A concordance index of 0.848 , representing predictive accuracy, was found upon internal validation of the nomogram. Our nomogram was superior in predictive accuracy to that with diagnostic PSA data, increasing the area under the curve (AUC) from $79.7 \%$ to $84.8 \%$ (Figure 3).

\section{DISCUSSION}

To our knowledge, this study is the first to examine the accuracy of a nomogram for the prediction of the prostate cancer risk in Chinese men, using a Chinese cohort for validation. We found that age, DRE, PSA and PV were independent predictors of prostate cancer risk. These findings are consistent with those in previous reports. ${ }^{6,7}$ However, after multivariate analysis, \% free PSA and TRUS findings were not independent predictors of a positive initial prostate biopsy. Previous studies have found $\%$ free PSA to be an independent predictor of prostate cancer; ${ }^{11,12}$ however, a report from Korea demonstrated that \% free PSA provided no added diagnostic benefit in Korean men from 50 to 65 years old with a PSA of $4.0-10.0 \mathrm{ng} \mathrm{ml}^{-1} .{ }^{13}$ Likewise, in our study cohort, $\%$ free PSA did not improve the accuracy of the nomogram for predicting a positive initial prostate biopsy. This discrepancy suggests that urologists in Asian and Western countries likely face different situations with regard to prostate cancer. Additionally, our findings were in agreement with those of Cavadas et al., ${ }^{7}$ who demonstrated that TRUS findings are not an independent predictor of prostate cancer. Conversely, Kawakami et al. ${ }^{14}$ reported that TRUS findings is an independent predictor of prostate cancer. In their study, they found that the accuracy of their nomogram for predicting prostate cancer was improved if TRUS findings were added.

There are a number of reasons why our findings differ from those of previous studies. First, the different study populations used in developing the models may result in substantially different findings because the characteristics of patients with prostate cancer in Western countries and Asian countries are different. ${ }^{6}$ To further evaluate the characteristics of our nomogram, we need to validate other nomograms using a Chinese population and compare the accuracy of our nomogram with others in subsequent studies. Additionally, the variables, especially the PSA range that was included in the prediction models, were very different from those found in other cohorts. In our model, we included age, PSA, PV, DRE status, \% free PSA and TRUS findings. Furthermore, all men, regardless of the PSA level, were included. Finally, different prostatic biopsy strategies at different

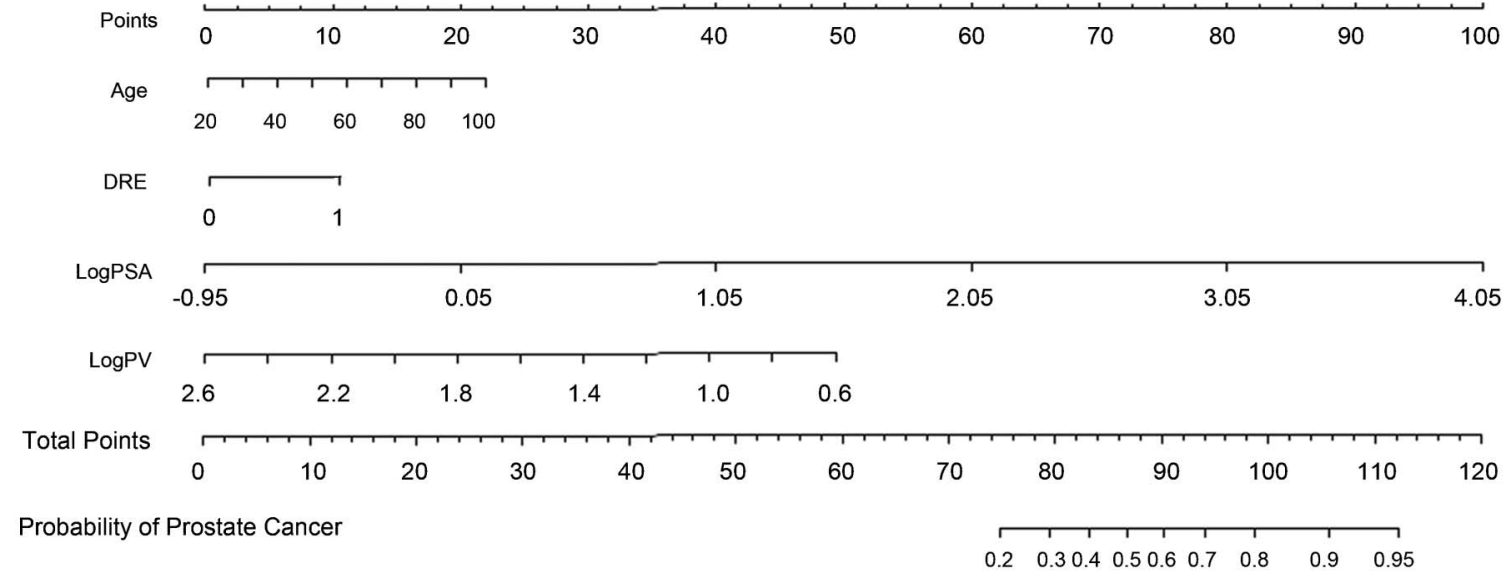

Figure 1 Nomogram for predicting a positive initial prostate biopsy. To determine the predicted probability of prostate cancer on initial biopsy, locate patient values on each axis. Draw a vertical line to the 'Point' axis to determine how many points are attributed to each variable. After summation of the points for all variables, locate the sum on the 'Total Points' line to determine the individual probability of prostate cancer on initial prostate biopsy on the 'Probability of Prostate Cancer' line. DRE, digital rectal examination ( $1=$ positive, $0=$ negative); PSA, prostate-specific antigen; $P V$, prostate volume. 


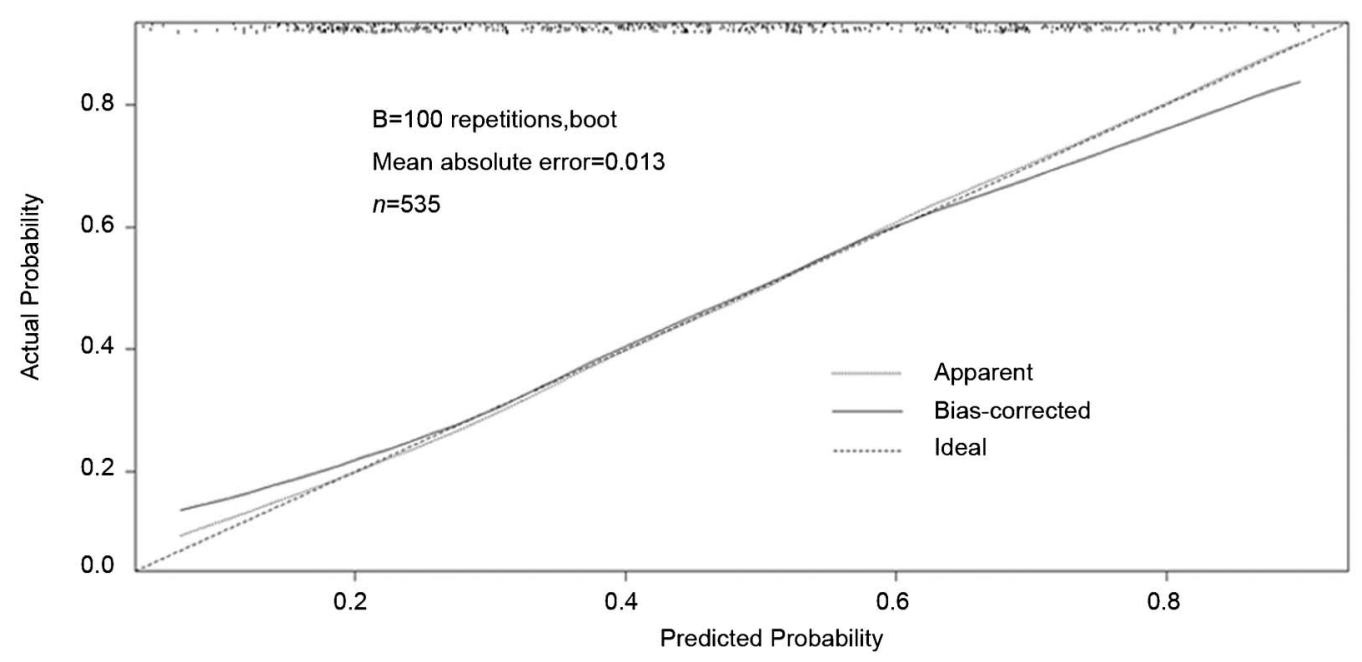

Figure 2 Result validation and predictive accuracy (84.8\%).

centres may lead to different prostate cancer detection rates, which could impact the results of the study. ${ }^{11}$

In the present study, we found that the accuracy of our nomogram for predicting a positive initial prostate biopsy was better than using PSA levels alone (AUCs were $84.8 \%$ and $79.7 \%$, respectively). While these results are similar with those reported from previous studies (Table 3), ${ }^{15-19}$ this is the first report to do so in a Chinese population, using a Chinese cohort. Recently, a systematic review comparing different models for predicting the risk of a positive prostate biopsy using PSA levels alone was reported. ${ }^{6}$ Twenty-three studies examining 36 models were included in this review. They found, with the exception of two studies, that all models had AUC values of 0.70 or greater, with eight reporting an AUC of $\geqslant 0.80$ and four reporting an AUC $\geqslant 0.85$. Fourteen studies compared the AUC with that with PSA levels alone, and all showed an added benefit from the added data, ranging from

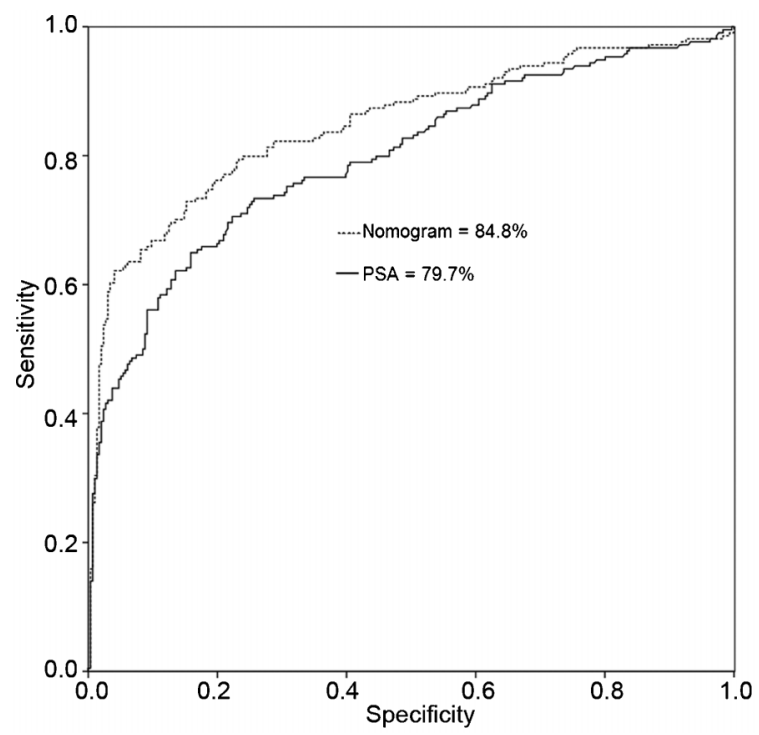

Figure 3 The area under the receiver-operating characteristic curve (AUC) for the nomogram and PSA alone for predicting a positive initial prostate biopsy. PSA, prostate-specific antigen.
0.02 to $0.26{ }^{6}$ Compared to reports in the literature, our model had a higher AUC and improved the predictive ability of PSA alone, increasing the AUC by $5.1 \%$ (from $79.7 \%$ to $84.8 \%$ ). In our study cohort, the majority of subjects $(90.8 \%)$ underwent 13 -core biopsies. This may have led to higher positive biopsy rates and improved the predictive accuracy of the nomogram. ${ }^{11}$ Furthermore, our nomogram was derived from a population based out of a Chinese tertiary hospital. The mean and median PSA values in our study cohort were extremely high because PSA testing is still not routinely used for early detection of prostate cancer in China; thus, the majority of prostate cancers are diagnosed at a later disease stage. PV, which was included in our predictive model, may also be a key factor in improving the accuracy of the nomogram. ${ }^{20}$

The present study is limited by the fact that nomograms for predicting a positive initial prostate biopsy are not new. However, many nomograms generated in Western countries may not be directly applicable to a Chinese population. Here we present the first nomogram for predicting a positive initial prostate biopsy developed using a completely Chinese population. The second limitation of our study is that it is retrospective. To confirm our findings, larger, prospective and randomized studies are warranted. Third, all the data in our study were obtained from a single, tertiary hospital, which may lead to biased results. The strategies used to diagnose prostate cancer may differ between centres, even those in a close geographical area. Our nomogram was derived from an unscreened cohort, and most men were diagnosed with late stage prostate cancer, which currently represents the majority of prostate cancer diagnoses in China. ${ }^{21,22}$ Finally, our nomogram has not been validated externally. Whether it can be universally applied to Chinese populations must be confirmed, and further studies are warranted.

\section{AUTHOR CONTRIBUTIONS}

$\mathrm{PT}, \mathrm{MU}$ and KJX conceived and designed the study. HC, XRD, YRL and BW collected the data. PT and WJY performed statistical analyses. $\mathrm{PT}$ and MU wrote the manuscript with input from all co-authors. All authors revised the manuscript and approved the final version.

\section{COMPETING FINANCIAL INTERESTS}

The authors declare no competing financial interests. 
Table 3 Predictive accuracy of various prostate cancer nomograms

\begin{tabular}{|c|c|c|c|c|c|c|c|c|c|}
\hline Study & $\mathrm{n}$ & Patients & Cancer rate (\%) & Validation & $\begin{array}{c}\text { Mean } \\
\text { age (year) }\end{array}$ & $\begin{array}{c}\text { Median PSA } \\
\left(n g m l^{-1}\right)\end{array}$ & $\begin{array}{c}\text { AUC for } \\
\text { model (\%) }\end{array}$ & $\begin{array}{l}A \cup C \text { for } \\
P S A(\%)\end{array}$ & $\begin{array}{c}\text { Increase in } \\
\text { AUC vs. PSA } \\
\text { alone (\%) }\end{array}$ \\
\hline $\mathrm{PCPT}^{15}$ & 5519 & CA $(95.6 \%)$ & 21.9 & Internal & $\geqslant 55$ & 1.5 & 70.2 & 67.8 & 2.4 \\
\hline Hernandez et al. ${ }^{16}$ & 1108 & CA (85.0\%) & 35.6 & NA & 63 & 5.4 & 66.7 & 61.9 & 4.8 \\
\hline Suzuki et al. ${ }^{17}$ & 834 & Japanese & 28.9 & NA & 70 & 13.1 (mean) & 81.8 & 69.8 & 12.0 \\
\hline Park et al. ${ }^{18}$ & 602 & Korean & 28.6 & NA & 66 & 6.77 & 72 & 66 & 6 \\
\hline Garzotto et al. ${ }^{19}$ & 1239 & CA $(93.5 \%)$ & 24.3 & Internal & 66 & 5.10 & 73 & 62 & 11 \\
\hline Our model & 535 & Chinese & 44.8 & Internal & 72 & 17.8 & 84.8 & 79.7 & 5.1 \\
\hline
\end{tabular}

Abbreviations: AUC, area under the curve; CA, Caucasian American; NA, not available; PCPT, Prostate Cancer Prevention Trial; PSA, prostate-specific antigen.

\section{ACKNOWLEDGMENTS}

This work was supported by the National Natural Science Foundation of China (No. 81072091/H1619), Guangdong Natural Science Foundation

(No. 10151006001000003) and Guangzhou Municipal Health Bureau Grant (No. 20121A021006) to PT.

1 Siegel R, Ward E, Brawley O, Jemal A. Cancer statistics, 2011: the impact of eliminating socioeconomic and racial disparities on premature cancer deaths. $C A$ Cancer J Clin 2011; 61:212-36.

2 Matsuda T, Saika K. Comparison of time trends in prostate cancer incidence (19732002) in Asia, from cancer incidence in five continents, Vols IV-IX. Jpn J Clin Oncol 2009; 39: 468-9.

3 Schroder FH, Hugosson J, Roobol MJ, Tammela TL, Ciatto S et al. Screening and prostate-cancer mortality in a randomized European study. N Engl J Med 2009; 360: 1320-8.

4 Andriole GL, Crawford ED, Grubb RL 3rd, Buys SS, Chia D et al. Mortality results from a randomized prostate-cancer screening trial. N Engl J Med 2009; 360: 1310-9.

5 Catalona WJ, Smith DS, Wolfert RL, Wang TJ, Rittenhouse HG et al. Evaluation of percentage of free serum prostate-specific antigen to improve specificity of prostate cancer screening. JAMA 1995; 274: 1214-20.

6 Schroder F, Kattan MW. The comparability of models for predicting the risk of a positive prostate biopsy with prostate-specific antigen alone: a systematic review. Eur Urol 2008; 54: 274-90.

7 Cavadas V, Osorio L, Sabell F, Teves F, Branco F et al. Prostate cancer prevention trial and European randomized study of screening for prostate cancer risk calculators: a performance comparison in a contemporary screened cohort. Eur Urol 2010; 58: 551-8.

8 Yoon DK, Park JY, Yoon S, Park MS, Moon DG et al. Can the prostate risk calculator based on western population be applied to asian population? Prostate $2011 ; 72: 721$ 9.

9 Tang P, Du W, Xie K, Fu J, Chen $\mathrm{H}$ et al. Characteristics of baseline PSA and PSA velocity in young men without prostate cancer: racial differences. Prostate 2012; 72: 173-80.

10 Zhu Y, Wang JY, Shen YJ, Dai B, Ma CG et al. External validation of the Prostate Cancer Prevention Trial and the European Randomized Study of Screening for Prostate Cancer risk calculators in a Chinese cohort. Asian J Androl 2012; 14: 738-44.
11 Chun FK, Briganti A, Graefen M, Montorsi F, Porter C et al. Development and externa validation of an extended 10-core biopsy nomogram. Eur Urol 2007; 52: 436-44.

12 Karakiewicz PI, Benayoun S, Kattan MW, Perrotte P, Valiquette L et al. Development and validation of a nomogram predicting the outcome of prostate biopsy based on patient age, digital rectal examination and serum prostate specific antigen. $J$ Urol 2005; 173: 1930-4.

13 Jeong IG, Lee KH. Percent free prostate specific antigen does not enhance the specificity of total prostate specific antigen for the detection of prostate cancer in Korean men 50 to 65 years old: a prospective multicenter study. J Urol 2008; 179 111-6.

14 Kawakami S, Numao N, Okubo Y, Koga F, Yamamoto S et al. Development, validation, and head-to-head comparison of logistic regression-based nomograms and artificial neural network models predicting prostate cancer on initial extended biopsy. Eur Uro 2008; 54: 601-11.

15 Thompson IM, Ankerst DP, Chi C, Goodman PJ, Tangen CM et al. Assessing prostate cancer risk: results from the Prostate Cancer Prevention Trial. J Natl Cancer Inst 2006; 98: 529-34.

16 Hernandez DJ, Han M, Humphreys EB, Mangold LA, Taneja SS et al. Predicting the outcome of prostate biopsy: comparison of a novel logistic regression-based model, the prostate cancer risk calculator, and prostate-specific antigen level alone. BJU Int 2009; 103: 609-14.

17 Suzuki H, Komiya A, Kamiya N, Imamoto T, Kawamura K et al. Development of a nomogram to predict probability of positive initial prostate biopsy among Japanese patients. Urology 2006; 67: 131-6.

18 Park JY, Yoon S, Park MS, Cho DY, Park HS et al. Initial biopsy outcome prediction in Korean patients-comparison of a noble web-based Korean prostate cancer risk calculator versus prostate-specific antigen testing. J Korean Med Sci 2011; 26 85-91.

19 Garzotto M, Hudson RG, Peters L, Hsieh YC, Barrera E et al. Predictive modeling for the presence of prostate carcinoma using clinical, laboratory, and ultrasound parameters in patients with prostate specific antigen levels $<$ or $=10 \mathrm{ng} / \mathrm{mL}$. Cancer 2003; 98: 1417-22.

20 Chun FK, Graefen M, Briganti A, Gallina A, Hopp J et al. Initial biopsy outcome prediction-head-to-head comparison of a logistic regression-based nomogram versus artificial neural network. Eur Urol 2007; 51: 1236-40.

21 Peyromaure EM, Mao K, Sun Y, Xia S, Jiang N et al. A comparative study of prostate cancer detection and management in China and in France. Can J Urol 2009; 16: 4472-7.

22 Zhang L, Wu S, Guo LR, Zhao XJ. Diagnostic strategies and the incidence of prostate cancer: reasons for the low reported incidence of prostate cancer in China. Asian $J$ Androl 2009; 11: 9-13. 